Appl i cat i on of Eddy-cur rent test i ng t echni que for hi gh- densi ty doubl e- I ayer pri nted ci rcuit boar d i nspect i on

\begin{tabular}{|c|c|}
\hline 著者 & $\begin{array}{l}\text { Chonsuwan K, Yanada Sot oshi, I wahar a } \\
\text { Masay oshi, Waki waka H., Shoj i S. }\end{array}$ \\
\hline $\begin{array}{l}\text { jour nal or } \\
\text { publ i cat i on title }\end{array}$ & $\begin{array}{l}\text { I NTERMAG ASI A 2005: Di gests of the I EEE } \\
\text { I nt er nat i onal Nagnet i cs Conf er ence }\end{array}$ \\
\hline page $r$ ange & $211-211$ \\
\hline year & $2005-01-01$ \\
\hline URL & ht t p: //hdl . handl e. net /2297/6912 \\
\hline
\end{tabular}




\title{
Application of Eddy-Current Testing Technique for High-Density Double-Layer Printed Circuit Board Inspection
}

\author{
K. Chomsuwan ${ }^{1}$, Student Member, IEEE, S. Yamada ${ }^{1}$, Member, IEEE, M. Iwahara $^{1}$, Member, IEEE, \\ H. Wakiwaka ${ }^{2}$ Member, IEEE, and S. Shoji ${ }^{3}$ \\ ${ }^{1}$ Institute of Nature and Environmental Technology, Kanazawa University, Ishikawa 920-8667, Japan \\ ${ }^{2}$ Electrical Engineering, Shinshu University, Nagano 380-8553, Japan \\ ${ }^{3}$ TDK Corporation, Nagano 385-8555, Japan
}

\begin{abstract}
High-density double-layer printed circuit board (PCB) inspection based on the eddy-current testing (ECT) technique is proposed in this paper. The ECT probe, which consisted of a planar meander exciting coil and spin-valve giant magnetoresistance (SV-GMR) sensor array, is used for this propose. Defects on both the top- and bottom-layer of the high-density double-layer PCB are examined by the ECT technique with scanning over either the top or bottom layer. The characteristics of the proposed ECT probe for high-density double-layer PCB inspection are studied. The inspection results of the high-density double-layer PCB model verify that applying the ECT technique enables identification of the defects of both the top and bottom layer with one-side scanning.
\end{abstract}

Index Terms-Eddy-current testing (ECT), inspection, printed circuit board (PCB), spin-valve giant magnetoresistance (SV-GMR).

\section{INTRODUCTION}

A N EDDY-CURRENT testing (ECT) probe, which consisted of a planar meander coil serving as an exciting coil and a spin-valve giant magnetoresistance (SV-GMR) device serving as a magnetic sensor, has been successfully applied to single-layer printed circuit board (PCB) inspection [1], [2]. The advantages of an SV-GMR sensor, such as high spatial resolution and high sensitivity to low magnetic field, are useful for inspection of a defect on a PCB conductor, as denoted in [1]-[3].

High-density double-layer PCB inspection based on a general method such as an optical method by a CCD camera has to examine both the top and bottom layer to obtain complete inspection. In this paper, inspection of a high-density double-layer PCB by an ETC probe composed of a planar meander coil and SV-GMR sensor array is proposed. Defects occurring on both the top and bottom layer of the PCB can be identified by scanning only over the top layer. The characteristics of the ECT probe for inspection of a defect on the PCB conductor and the effect of distance between the sensing level and PCB conductor (lift-off height) are investigated to verify that the probe is able to inspect defects on both the top and bottom layer of high-density double-layer PCB. In addition, the inspection of the high-density double-layer PCB model is demonstrated.

\section{SYSTEM CONFIGURATION}

\section{A. Proposed ECT Probe Structure}

The ECT probe structure and small-signal characteristics of the SV-GMR sensor are shown in Fig. 1(a) and (b). The probe consists of a planar meander coil serving as an exciter and an SV-GMR sensor array consisting of three SV-GMR elements. The SV-GMR sensor array was mounted on the planar meander coil sandwiched by two films to separate the coil from the

Digital Object Identifier 10.1109/TMAG.2005.855173

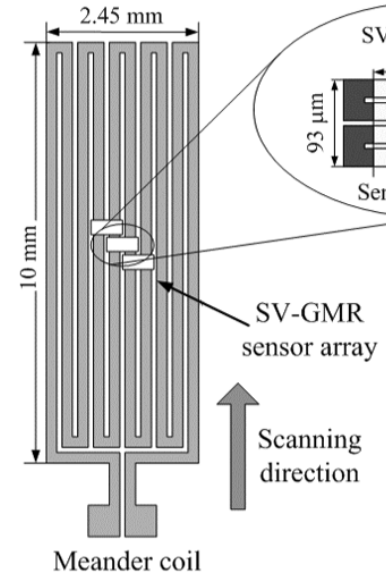

SV-GMR senso $100 \mu \mathrm{m}$

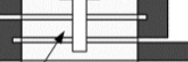

Sensing axi

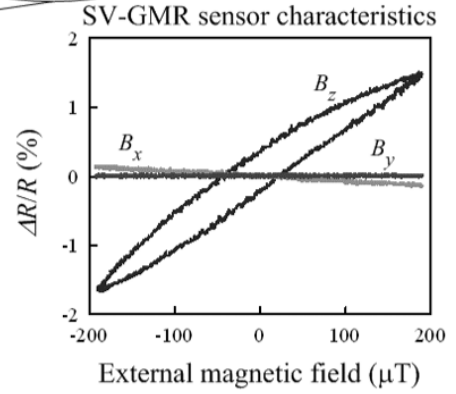

(a)

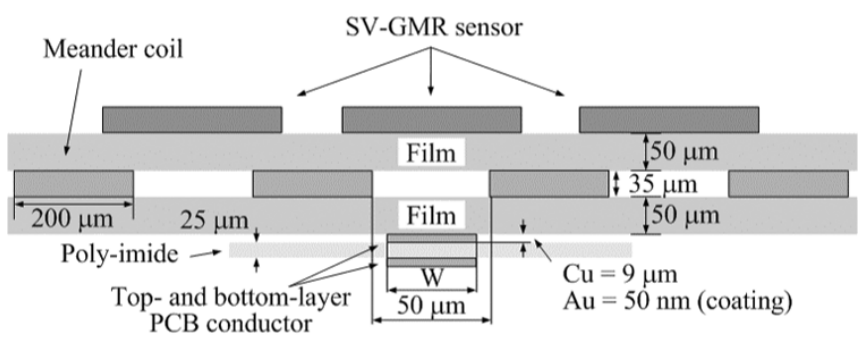

(b)

Fig. 1. Proposed ECT probe structure and SV-GMR sensor characteristics: (a) Top view of probe and SV-GMR sensor characteristics and (b) cross section of probe.

SV-GMR sensor array and the PCB conductors. Therefore, the distance from the SV-GMR surface to the PCB conductor or lift-off height is $135 \mu \mathrm{m}$. A high-frequency excitation current was fed to a planar meander coil to generate eddy currents flowing in a PCB conductor. The magnetic field $B_{z}$ usually occurs at the defect point or PCB conductor boundary that 


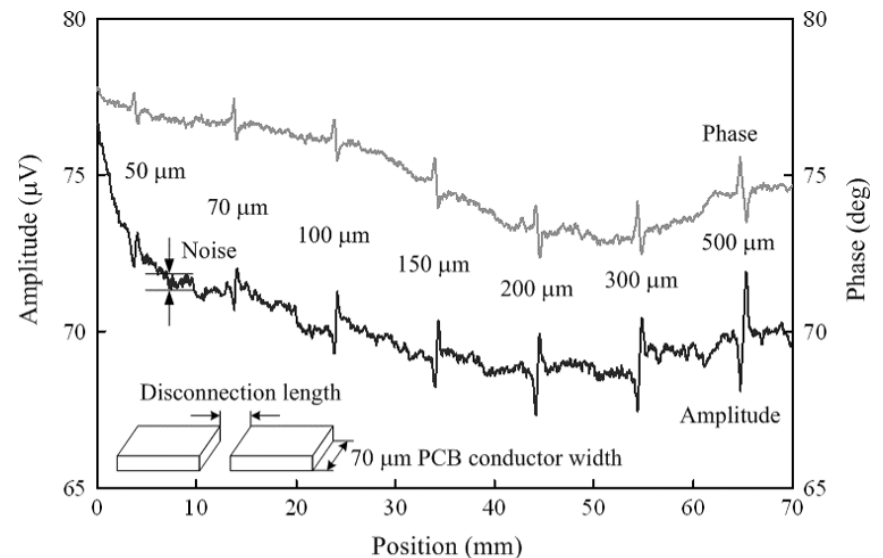

Fig. 2. Amplitude and phase of ECT signal obtained from scanning over PCB conductor with $70-\mu \mathrm{m}$ width.

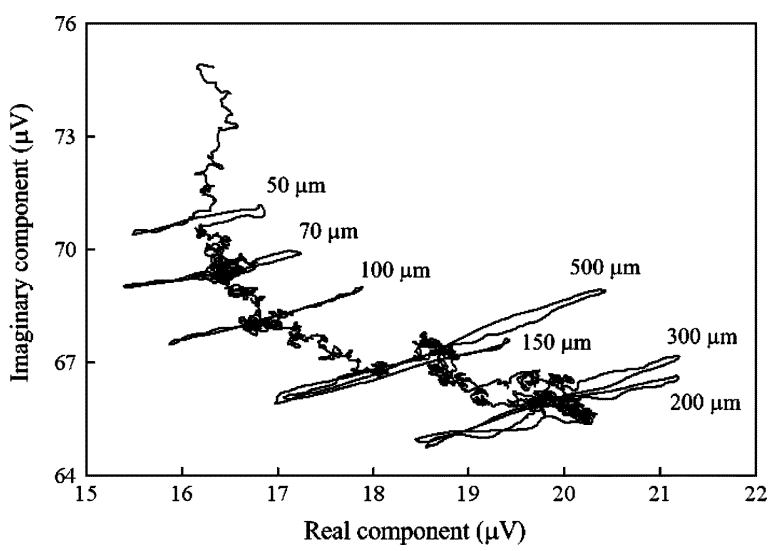

Fig. 3. Cosine (real) and sine (imaginary) component of ECT signal in Fig. 2 plotted on the complex plane.

is perpendicular to the scanning direction. Sensing directions of the SV-GMR sensor array were set to detect the magnetic field $B_{z}$ that is parallel to the scanning direction. Each of the SV-GMR sensors consists of four strips, and each strip has a dimension of $100 \mu \mathrm{m} \times 18 \mu \mathrm{m}$. Therefore, the total effective area of each of SV-GMR sensor is $100 \mu \mathrm{m} \times 93 \mu \mathrm{m}$ with a $7-\mu \mathrm{m}$ gap between strips. The normal resistance of the SV-GMR sensor is around $400 \Omega$. The SV-GMR sensor sensitivity in the sensing axis is around $8.4 \% / \mathrm{mT}$, whereas it is lower than $1 \% / \mathrm{mT}$ in the other axes.

\section{B. Experimental Setup}

The sinusoidal current of $200 \mathrm{~mA}$ at a frequency of $5 \mathrm{MHz}$ was fed to the planar meander coil. A constant dc current of 5 $\mathrm{mA}$ was also fed to the SV-GMR sensor. A lock-in amplifier was used to measure the voltage drop at the SV-GMR sensor. From the SV-GMR sensor dimension, the scanning pitch was set at $20 \mu \mathrm{m}$.

\section{EXPERIMENTAL RESULTS}

\section{A. Detection of Defect on PCB Conductor by the Proposed ECT Probe}

The PCB model with 9- $\mu$ m PCB conductor thickness made from $\mathrm{Cu}$ coated by $0.05-\mu \mathrm{m} \mathrm{Au}$ was used in the experiment. Two kinds of defect were allocated on the model to study the characteristics of the ECT probe for PCB inspection. The first

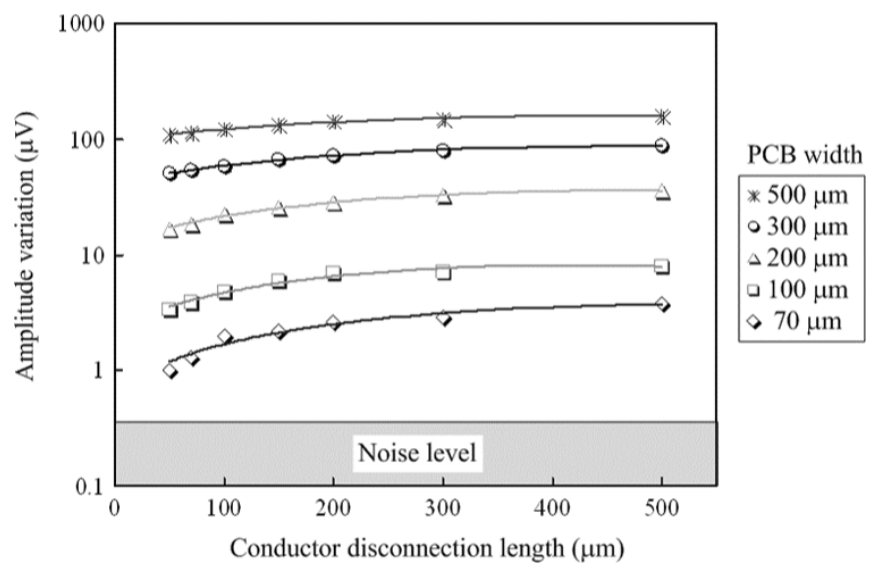

Fig. 4. ECT signal variations against conductor disconnection length.

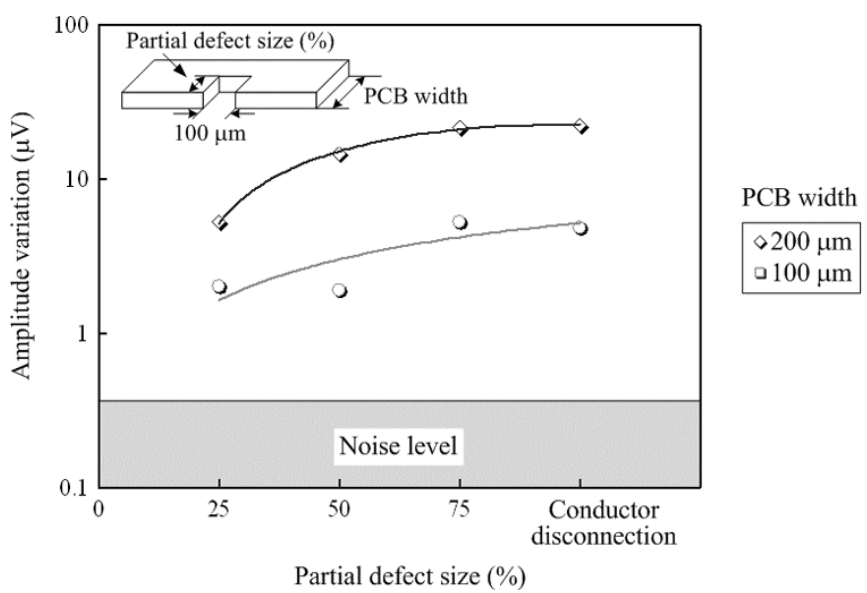

Fig. 5. ECT signal variations against partial defects on PCB conductor track widths.

is conductor disconnections, and the second is partial defects on the track width of the PCB conductor.

As shown in Fig. 2, the SV-GMR sensor can detect the magnetic field variation at defect points and provides variation of signals, both amplitude and phase. The complex plane in Fig. 3 conveniently represents the variation of signals, both amplitude and phase, at defect points as a real and imaginary component. The variations of signals are directly proportional to the defect size, and they also depend on conductor width, whereas the noise signals, defined in Fig. 2, are constant with less than 0.6 $\mu \mathrm{V}$, as shown in Fig. 4. The signal is larger than noise, around two times, although the thin PCB conductor with $70-\mu \mathrm{m}$ width and 50- $\mu \mathrm{m}$ disconnection length is inspected. Inspection of partial defects on the PCB track width is also performed by the proposed ECT probe, as shown in Fig. 5. The partial defect has the effect of decreasing the signal variation as compared with the signal variation in the case of conductor disconnection.

\section{B. Lift-Off Height Effect}

The distance between PCB conductors and sensing level is very important for the inspection of bottom-layer PCB conductor. Fig. 6 shows the inspection results obtained from scanning over the PCB conductor with $100 \mu \mathrm{m}$ width. Conductor disconnections ranging from 500 to $50 \mu \mathrm{m}$ were allocated on the PCB conductor. The results show that the proposed ECT probe is able to inspect the defects on the PCB conductor with $235-\mu \mathrm{m}$ lift-off height, although the signal variations are very 


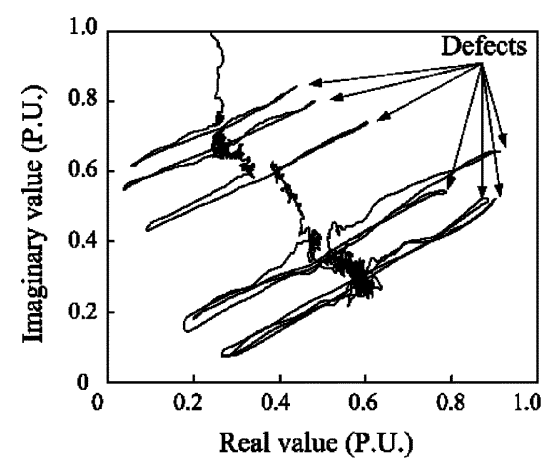

(a)

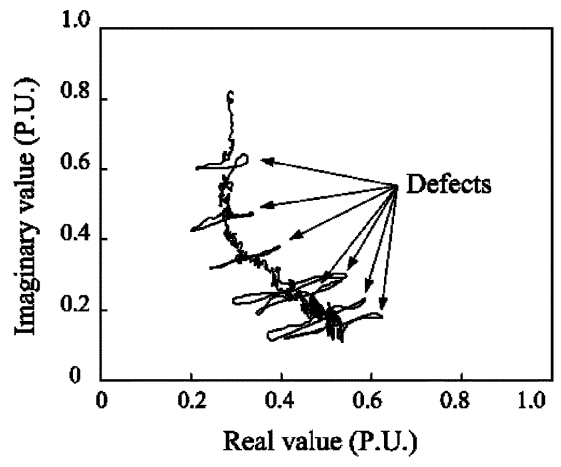

(b)

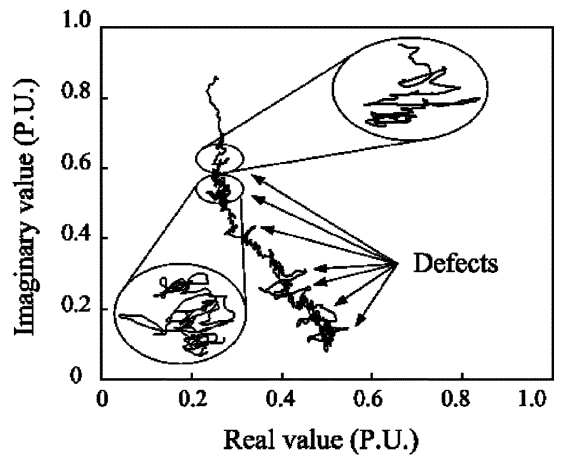

(c)

Fig. 6. Complex plane of ECT signal obtained from scanning over a PCB conductor, $100 \mu \mathrm{m}$ width, with different distances from sensing level to PCB conductor: (a) Lift-off height $=135 \mu \mathrm{m}$; (b) lift-off height $=185 \mu \mathrm{m}$; and (c) lift-off height $=235 \mu \mathrm{m}$.

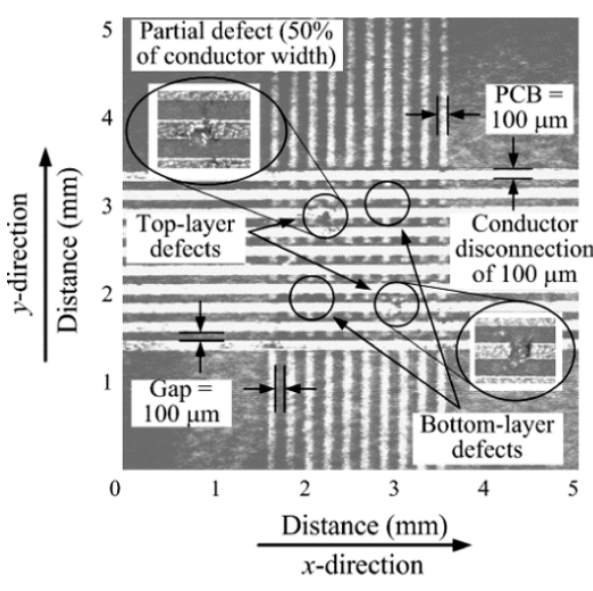

(a)

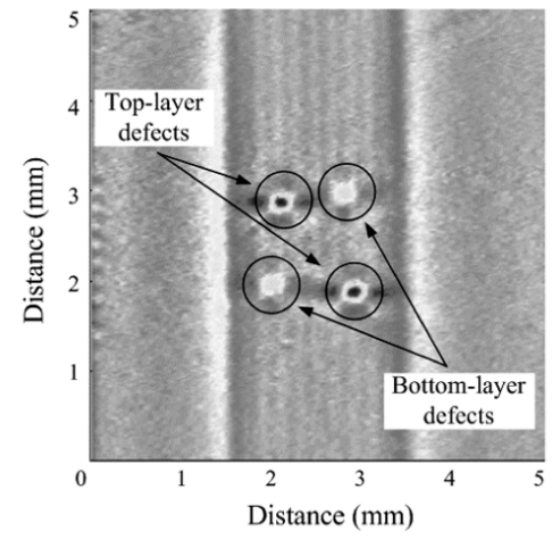

(b)

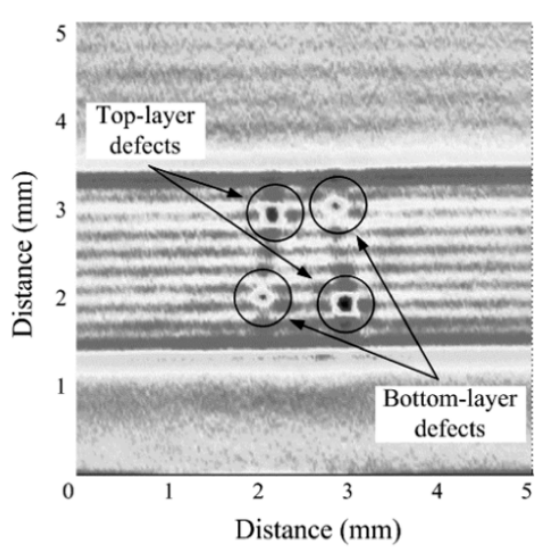

(c)

Fig. 7. High-density double-layer PCB model with defect points (100- $\mu \mathrm{m}$ disconnection length) and its inspection results obtained from the proposed probe: (a) High-density double-layer PCB model; (b) $x$-direction scanning; and (c) $y$-direction scanning.

small. This means that the probe is capable of inspecting the defects at the bottom layer if the distance between PCB conductor and sensing level is not over $200 \mu \mathrm{m}$.

\section{Example of High-Density Double-Layer PCB Inspection}

A high-density double-layer PCB with a dimension of $5 \mathrm{~mm} \times 5 \mathrm{~mm}$, as shown in Fig. 7(a), was used as a model. The PCB conductors parallel to the $x$ direction are the top-layer conductor, and the others are the bottom-layer conductor. The disconnection and partial defects are also allocated on both the top and the bottom layer of the PCB model.

Two-dimensional (2-D) images reconstructed from the ECT signal obtained from scanning over the top layer of the PCB model in the $x$ and $y$ direction are shown in Fig. 7(b) and (c), respectively. The numerical gradient technique is a simple image processing technique used to eliminate signal offset and enhance the signal at the defect points. The 2-D images show that the probe is capable of inspecting the defect clearly, although the defect points are allocated on a bottom-layer PCB conductor.

\section{CONCLUSION}

Inspection of a high-density double-layer printed circuit board (PCB) based on the eddy-current testing (ECT) technique with spin-valve giant magnetoresistance (SV-GMR) sensor array was proposed. The results show that applying the ECT technique enables identification of a microcrack on the high-density double-layer PCB. The main disadvantage of this system is a long inspection time, although the SV-GMR sensor array is already used. Therefore, the usage of the sensor matrix will be useful for improving inspection time.

\section{ACKNOWLEDGMENT}

This work was supported in part by the Japan Society for Promotion of Science (Category B, 14350218) under a Grant-in-Aid for Scientific Research.

\section{REFERENCES}

[1] S. Yamada, K. Chomsuwan, Y. Fukuda, M. Iwahara, H. Wakiwaka, and S. Shoji, "Eddy-current testing probe with spin-valve type GMR sensor for printed circuit board inspection," IEEE Trans. Magn., vol. 40, no. 4, pp. 2676-2678, Jul. 2004.

[2] K. Chomsuwan, Y. Fukuda, S. Yamada, M. Iwahara, H. Wakiwaka, and S. Shoji, "GMR sensor utilization for PCB inspection based on the eddycurrent testing technique," Trans. Magn. Soc. Jpn., vol. 4, pp. 39-42, Feb. 2004.

[3] T. Dogaru and S. Smith, "Giant magnetoresistance-based eddy-current sensor," IEEE Trans. Magn., vol. 37, no. 5, pp. 3831-3838, Sep. 2001.

Manuscript received February 1, 2005. 